\title{
Feeding Honeybee Colonies with Honeybee-Specific Lactic Acid Bacteria (Hbs-LAB) Does Not Affect Colony-Level Hbs-LAB Composition or Paenibacillus larvae Spore Levels, Although American Foulbrood Affected Colonies Harbor a More Diverse Hbs-LAB Community
}

\author{
Sepideh Lamei ${ }^{1,2} \cdot$ Jörg G. Stephan ${ }^{1,3}$ (D) $\cdot$ Bo Nilson $^{4,5}$ (D) $\cdot$ Sander Sieuwerts $^{6}$ (D) Kristian Riesbeck $^{2}$ (D) \\ Joachim R. de Miranda ${ }^{1}$ (D) E Eva Forsgren ${ }^{1}$ (D)
}

Received: 12 April 2019 / Accepted: 26 August 2019 / Published online: 10 September 2019

(C) The Author(s) 2019

\begin{abstract}
The main current methods for controlling American Foulbrood (AFB) in honeybees, caused by the bacterial pathogen Paenibacillus larvae, are enforced incineration or prophylactic antibiotic treatment, neither of which is fully satisfactory. This has led to an increased interest in the natural relationships between the pathogenic and mutualistic microorganisms of the honeybee microbiome, in particular, the antagonistic effects of Honeybee-Specific Lactic Acid Bacteria (hbs-LAB) against $P$. larvae. We investigated whether supplemental administration of these bacteria affected $P$. larvae infection at colony level over an entire flowering season. Over the season, the supplements affected neither colony-level hbs-LAB composition nor naturally subclinical or clinical $P$. larvae spore levels. The composition of hbs-LAB in colonies was, however, more diverse in apiaries with a history of clinical AFB, although this was also unrelated to P. larvae spore levels. During the experiments, we also showed that qPCR could detect a wider range of hbs-LAB, with higher specificity and sensitivity than mass spectrometry. Honeybee colonies are complex super-organisms where social immune defenses, natural homeostatic mechanisms, and microbiome diversity and function play a major role in disease resistance. This means that observations made at the individual bee level cannot be simply extrapolated to infer similar effects at colony level. Although individual laboratory larval assays have clearly demonstrated the antagonistic effects of hbs-LAB on $P$. larvae infection, the results from the experiments presented here indicate that direct conversion of such practice to colony-level administration of live hbs-LAB is not effective.
\end{abstract}

Keywords American foulbrood · Apis mellifera $\cdot$ Beneficial microbes $\cdot$ Bifidobacterium $\cdot$ Intestinal microbiota $\cdot$ Lactobacillus

Electronic supplementary material The online version of this article (https://doi.org/10.1007/s00248-019-01434-3) contains supplementary material, which is available to authorized users.

Jörg G. Stephan

jorg.stephan@slu.se

1 Department of Ecology, Swedish University of Agricultural Sciences, Uppsala, Sweden

2 Clinical Microbiology, Department of Translational Medicine, Lund University, Malmö, Sweden

3 Swedish Species Information Centre, Swedish University of Agricultural Sciences, Uppsala, Sweden

4 Clinical Microbiology, Labmedicine, Region Skåne, Lund, Sweden

5 Department of Laboratory Medicine Lund, Lund University, Lund, Sweden

6 Arla Innovation Center, Aarhus, Denmark

\section{Introduction}

Apis mellifera is a social insect with a diverse microbiota consisting of dozens of bacterial taxa, including Grampositive and Gram-negative bacteria as well as alpha-, beta-, and gamma-proteobacteria. Most of these bacteria are not pathogenic, with a few critical exceptions [1-3]. One of these is the spore-forming bacterium Paenibacillus larvae, the causative agent of American foulbrood (AFB) which is the most lethal brood disease of honeybees, and ultimately fatal to the colony if left untreated [4]. Different $P$. larvae genotypes have been identified, with slightly different pathological consequences at individual and colony level $[5,6]$. P. larvae spores germinate in the midgut of young larvae, after which the bacteria breach the intestinal lining and invade the larval tissues, where they continue to proliferate and ultimately contract into 
billions of spores when the larval tissues are exhausted. These larval remains dry into sticky, hard scales that bees have difficulty removing and thus constitute a continuous source of new infections in successive brood cycles. This perpetual reinfection, the distribution of the spores throughout the colony by young cleaning and nursing bees, together with the hardiness and viability of the spores, are the key factors driving the lethality and within-colony epidemiology of AFB $[4,7]$. The between-colony epidemiology is driven primarily by beekeepers moving contaminated material between colonies, and at a more local scale by drifting, swarming, and robbing bees $[8,9]$.

AFB is controlled primarily by burning symptomatic colonies, thereby destroying the long-lived spores residing in the frames and hive material, although the bees can sometimes be saved as an artificial swarm, housed on new material [4]. An alternative approach, popular in North America but illegal in Europe, is to target the germinating bacteria with antibiotics such as oxytetracycline and tylosin, thereby suppressing AFB symptoms without addressing the principal cause of the disease, i.e., the persistence of the spores. Prophylactic use of these antibiotics has inevitably led to antibiotic resistance in P. larvae $[10,11]$. However, the strong antagonistic effects of naturally occurring lactic acid bacteria in honeybees on the infectivity and pathogenicity of $P$. larvae has identified a possible new approach to AFB control [12-15]. Beneficial bacteria belonging to the Lactobacilli and Bifidobacteria have been shown to promote honeybee health through activating the honeybee's immune defenses $[14,16]$, producing antimicrobial compounds inhibiting bacterial competitors [17-19], and by outcompeting pathogenic bacteria $[2,12,13]$.

The honeybee-specific Lactic Acid Bacteria (hbs-LAB) comprise a group of functionally similar bacteria that reside primarily in the honey crop [20]. Thirteen genetically distinct lactic-acid producing bacteria have been identified from the honeybee crop, of which nine are Lactobacilli and four are Bifidobacteria $[20,21]$. Between then, they produce a range of metabolites, such as organic acids [22], extracellular proteins, lipopolysaccharides, and lipoteichoic acid [23] that may contribute to their inhibitory effect on $P$. larvae in microbial and infection bioassays $[18,19]$.

However, the honeybee colony is a complex superorganism with both individual and social immune defenses that work in tandem for managing overall colony health but lead to different consequences for infected individuals. Beneficial health effects at individual bee level do not therefore axiomatically translate into similar health benefits at colony level [24-27]. The evidence for any health benefits of manipulating the honeybee microbiome at colony level is mixed, with some studies showing largely negative effects [28], while others show more positive effects $[12,13]$. We previously demonstrated the inhibitory effects of hbs-LAB on P. larvae infection in individual larvae [18] and that this included the secretions of antimicrobial substances that inhibit the germination of $P$. larvae spores and vegetative cell growth [29]. However, we also showed that this type of hbs-LAB formulation and administration was not effective at colony level as a curative agent for AFB-diseased colonies, since neither $P$. larvae spore levels nor AFB disease symptoms were affected throughout a whole bee season [30].

The aim of the present study was therefore to evaluate whether hbs-LAB supplements could instead function as a preventative agent, by lowering the background concentrations of $P$. larvae spores in naturally subclinically infected colonies, in apiaries with a history of AFB. A secondary aim was to characterize the natural hbs-LAB composition in colonies from apiaries with sub-clinical levels of $P$. larvae, relative to colonies in newly established "clean" apiaries, and whether supplemental administration of hbs-LAB affected the natural hbs-LAB composition in both types of colonies and apiaries. We furthermore aimed to discover a link between (cumulative) abundance and diversity of hbs-LAB and $P$. larvae abundance at colony level. The composition and amount of hbs-LAB was extrapolated from the doseresponse experiments at individual larvae levels [18, 31]. Lastly, we compared the relative efficacy and reliability of two major methods for the detection and identification of hbs-LAB: Matrix-Assisted Laser Desorption/Ionization Time-of-Flight Mass Spectrometry (MALDI-TOF MS) and qPCR. This was done for a subset of eight hbs-LAB strains that could be detected by both methods.

\section{Methods}

\section{Experimental Design}

The experiment was performed in four honeybee apiaries located around the county Uppland with 12 colonies per apiary, all under standard commercial management. Two of the apiaries have had a history of persistent AFB (clinical outbreaks in 2012 and 2013) in all cases caused by P. larvae of ERIC I genotype [32]). The colonies in these apiaries were placed in quarantine management, with completely separated equipment from the remaining part of the beekeeping operation. The other two apiaries were established in 2015, with artificial swarms introduced on completely new hives and frame material and managed separately from the quarantined apiaries, with $>10 \mathrm{~km}$ pairwise distance between all of the apiaries, on a safe distance outside the normal flight range of honeybees. All bees and queens in the experiments were derived from the same stock and beekeeping operation. In the experiments and analyses, these two sets of apiaries and their colonies are referred to as "quarantined" and "non-quarantined," respectively. Prior to the start of the experiment in 2016, the P. larvae status of all 48 colonies in the four apiaries was 
determined from adult bee samples collected during autumn 2015, using standard P. larvae spore cultivation methods [33], since spore counts from adult bee samples are the most reliable predictor of AFB disease symptoms [34]. The samples from the quarantined apiaries had between 100 and 40,000 $P$. larvae spores per honeybee while the samples from the non-quarantined apiaries were all free of $P$. larvae spores. During spring 2016, six colonies in each of the four apiaries were administered hbs-LAB supplements while the other six were given placebo supplements, in a double-blinded fashion. The supplements were administered on two occasions, in April and May 2016, coincident with the first and second sampling occasions.

\section{Composition of Hbs-LAB Supplements}

The composition and total amount of bacteria in the hbs-LAB supplement to be used at colony level $\left(10^{6}\right.$ colony forming units (CFU)/colony) was extrapolated from previous doseresponse research at individual level, in laboratory bioassays, through multiplying the optimum per-larva dose (2500 CFU/ larva) [18] by the expected number of first instar larvae in a standard colony in April-May in Sweden (approximately 400). All hbs-LAB, including nine Lactobacillus spp. (Lactobacillus kunkeei Fhon2N, Lactobacillus apinorum Fhon13N, Lactobacillus mellis Hon2N, L. mellifer Bin4N, L. apis Hma11N, Lactobacillus helsingborgensis Bma5N, Lactobacillus melliventris Hma8N, Lactobacillus kimbladii Hma2N, Lactobacillus kullabergensis Biut2N) and four species/major strains of Bifidobacteria (Bifidobacteria asteroides $\mathrm{Bin} 2 \mathrm{~N}, B$. asteroides $\mathrm{Bin} 7 \mathrm{~N}, B$. asteroides Hma3N and Bifidobacteria coryneforme Bma6N) [20, 21], were incubated individually and anaerobically at $35^{\circ} \mathrm{C}$ in Man, Rogosa \& Sharpe (MRS; Oxoid, England) broth, supplemented with 2\% fructose (Merck, Sollentuna, Sweden) and $0.1 \%$ L-cysteine (Sigma-Aldrich, Stockholm, Sweden) [35]. A $30-\mathrm{mL}$ mixture of all $13 \mathrm{hbs}-\mathrm{LAB}$ was prepared from equal volumes of each individual bacterial strain at $10^{7} \mathrm{CFU} / \mathrm{mL}$, and was subsequently freeze-dried in ten aliquots. Prior to feeding, the freeze-dried bacteria were resuspended in 1:10 volumes of water and incubated overnight at $35^{\circ} \mathrm{C}$, resulting in $300 \mu \mathrm{L}$ bacterial suspension at $7.7 \times 10^{6} \mathrm{CFU} / \mathrm{mL}$ for each aliquot. Each bacterial aliquot was added to $1.5 \mathrm{~kg}$ inverted sugar solution (Bifor®, Nordic sugar, Germany) diluted with $1 \mathrm{~L}$ warm water $\left(45^{\circ} \mathrm{C}\right)$. This mixture was incubated at 30 $40{ }^{\circ} \mathrm{C}$ overnight and $10^{6} \mathrm{CFU}$ (approximately $73 \mathrm{~mL}$ ) was administered to each honeybee colony.

\section{Sample Management}

Adult honeybees were sampled monthly from each colony between April 15 and September 15, 2016, resulting in six sampling occasions. Approximately 200 adult honeybees per sample were collected from the brood chamber in a small cardboard box. Additionally, for each colony, 20 honey crops with nectar were pulled from worker bees (see within [36]) and pooled in a $1.5-\mathrm{mL}$ microcentrifuge tube containing $1 \mathrm{~mL}$ sterile physiological saline solution $(0.9 \%)$. The honeybee samples and honey crops were stored at $-20{ }^{\circ} \mathrm{C}$ until bacterial analysis. Paenibacillus larvae spores are highly resistant and their viability is unaffected by freezing at $-20{ }^{\circ} \mathrm{C}$ [37].

\section{Cultivation of $P$. larvae}

Worker honeybee samples were homogenized and cultivated on MYPGP-agar plates as described by Forsgren and Laugen [38]. Bacterial colonies were confirmed by PCR as described previously [39]. The number of $P$. larvae CFU was multiplied by the various dilution factors in the procedure to estimate the number of $P$. larvae $\mathrm{CFU}$ per honeybee prior to data analysis.

\section{Analysis of Hbs-LAB Composition in Honey Crops}

The number and composition of hbs-LAB in the honey crops was assessed with two complementary methods: quantitative PCR (qPCR) and Matrix-Assisted Laser Desorption/ Ionization Time-of-Flight (MALDI-TOF) Mass Spectrometry (MS). The 20 honey crops from each colony sample were homogenized in $1 \mathrm{~mL}$ bee physiological saline using a single-use tip. These homogenates were thereafter characterized directly by qPCR-based detection and quantification of the species/strain-specific hbs-LAB DNA in the honeybee crop, and indirectly by MALDI-TOF MS analysis of individual bacterial colonies cultured from the homogenates (see below).

\section{Quantitative PCR (qPCR)}

DNA was extracted from $100 \mu \mathrm{L}$ of honeycrop homogenate using the DNeasy Blood and Tissue kit (Qiagen, Germany), with minor modifications of the manufacturer's protocol as described previously [35]. The DNA of the different hbsLAB was quantified by qPCR using a modified version of Nadkarni et al. [40], using proprietary primers developed by ConCellae AB (Lund, Sweden) based on selective gene sequences to allow the specific detection of each of the hbs-LAB species and strains investigated here. The performance characteristics of the different assays, including detection limits, can also be obtained from ConCellae AB. The PCR reaction was performed in a total volume of $10 \mu \mathrm{L}$ using $5 \mu \mathrm{L}$ of Applied Biosystems SYBR green PCR master mix (Thermo Fisher Scientific, UK), $0.3 \mu \mathrm{M}$ each of the forward and reverse primers for each hbs-LAB species and strain, and, finally, $4 \mu \mathrm{L}$ of DNA template (10-50 ng/ $\mu \mathrm{L})$. A negative control containing water instead of DNA template was included in each run. Amplification and detection of DNA by qPCR were 
performed with the Quantstudio 7 Flex System (Applied Biosystems, Thermo Fisher Scientific, MA, USA) using optical-grade 384-well plates. The reaction conditions for amplification of DNA were $50^{\circ} \mathrm{C}$ for $2 \mathrm{~min}, 95^{\circ} \mathrm{C}$ for $2 \mathrm{~min}$ and 40 cycles of $95^{\circ} \mathrm{C}$ for $15 \mathrm{~s}$ and $60^{\circ} \mathrm{C}$ for $1 \mathrm{~min}$. For each hbs$\mathrm{LAB}$, the absolute number of bacterial genomes, as represented by the specific gene PCRs, was calculated by the Quantstudio Software version 1.2 supplied by Applied Biosystems, using external calibration curves based on tenfold serial dilutions of DNA extracted from known amounts (in $\mathrm{CFU}$ ) of pure culture of each hbs-LAB. The data was multiplied by the various dilution factors in the experiments to determine the estimated CFU for each hbs-LAB in each sample prior to data analysis.

\section{Matrix-Assisted Laser Desorption/Ionization Time-of-Flight (MALDI-TOF) Mass Spectrometry (MS)}

Bacteria in the honey crop homogenates were cultured in tenfold serial dilutions on MRS agar supplemented with $2 \%$ fructose (Merck, Sollentuna, Sweden) and 0.1\% L-cysteine (Sigma-Aldrich, Sweden) [31,35]. Fifteen individual bacterial colonies from each sample were transferred to a 96-well steel target plate (Bruker Daltonics, Sweden) and covered with $1 \mu \mathrm{L} 70 \%$ formic acid and $1 \mu \mathrm{L}$ of $10 \mathrm{mg} / \mathrm{mL} \alpha$-cyano 4 hydroxycinnamic acid (HCCA) in a matrix solution (SigmaAldrich), as described previously [29]. The MS of each bacterial colony was determined by MALDI-TOF MS [41], analyzed using a Microflex instrument, the FlexControl and MALDI Biotyper 3.1 software with MBT Compass library, DB-6903 MSP (Bruker Daltonics). The resulting MS profiles of the bacterial colonies were analyzed against a combined library consisting of the MBT Compass library and an inhouse reference database for the different hbs-LAB (HBSLAB database) [21], to allow the hbs-LAB identification of each bacterial colony. A score of 2.0 or greater was needed for the positive hbs-LAB identification.

\section{Data Analysis}

The raw data consisted of quantitative measures for the CFU per bee for $P$. larvae (determined by culturing), CFU per sample for each of the $10 \mathrm{hbs}$-LAB (determined by qPCR), and the qualitative hbs-LAB identification of individual cultured bacterial colonies (determined by MALDI-TOF MS) present in each of the 48 honeybee colonies on seven sampling occasions before, during, and after treatment with supplemental hbs-LAB or placebo. The MALDI-TOF MS data have a Bernoulli distribution (detected, not detected), whereas the P. larvae spore counts and $\mathrm{qPCR}$ have a Poisson distribution. Furthermore, the qPCR data of hbs-LAB were compressed into a Shannon diversity index capturing the strain richness (number of strains detected) and abundance of the ten hbsLAB.

The primary objectives of the analyses were to test the effect of treatment (oral administration of hbs-LAB) and the quarantine status of the apiary on the spore counts of $P$. larvae and the presence, abundance, and diversity of hbs-LAB in the colonies. These two main objectives were pursued using the qPCR data as it offered a measure of abundance for each hbsLAB. Although the categorization of "quarantined" and "nonquarantined" colonies was based on AFB history of the apiaries, supported by $P$. larvae spore analyses of the 2015 preexperiment samples, the 2016 experimental samples also identified low levels of $P$. larvae spores in ten of the 135 samples from the non-quarantined colonies (Fig. S1). We have no simple explanation for these observations, other than that the $P$. larvae detection in the non-quarantined colonies may simply reflect random probabilistic detection out of a natural, low background presence. However, these findings mean that the quarantine status of a colony, or an apiary, is by itself not sufficient as an explanatory variable in the data analysis. We therefore used $P$. larvae spore counts as the primary explanatory variable for explaining hbs-LAB diversity (Table 1: Model 1; hereafter M1) or cumulative hbs-LAB abundance (Table 1: M2) in the honey crop. The main function of the quarantine/non-quarantine categories is to represent other systematic differences between the colonies in these apiaries, which consists principally of the age of the equipment (new in the non-quarantined apiaries, used in the quarantined apiaries) and secondarily of the developmental background of the colonies in 2015 (artificial swarms in the non-quarantined apiaries, established colonies in the quarantined apiaries). As recommended by Schielzeth et al. [42], the only continuous explanatory variable ("Diversity", as represented by the Shannon index) was centered and scaled.

The second series of analysis focused on the reverse effect, that of $P$. larvae spore level on hbs-LAB abundance. This was tested with a multivariate analysis of co-variance (MANCOVA) with all ten hbs-LAB as response variable (Table 2: M3). Before the backward selection, the multivariate normality of residuals was checked graphically and homogeneity of covariance matrices was checked with the Box's Mtest [43]. To meet the requirements of multivariate normality, we transformed the response variables $(\log (x+1))$ and as there was indication of low homogeneity in the covariance matrices we used Pillai's trace [43]. To avoid an unnecessary complex model (e.g., indications of a temporal effect was not seen, see below) we did not include the hierarchical data structure here and used the abundances of each hbs-LAB regardless of sampling occasion and honeybee colony identity. After the multivariate model analysis, the univariate linear models for each individual hbs-LAB were extracted to check for each strain whether its individual abundance was affected by colony treatment and/or quarantine status. Furthermore, we 
Table 1 Analysis-of-deviance tables (Type III test) from generalized linear mixed models (GLMMs) investigating the effect of hbs-LAB diversity (M1) and cumulative abundance of all hbs-LAB (M2) on the P. larvae spore level. Non-significant terms (italicized) were removed stepwise from the final minimal adequate model starting from the bottom row. "” means latter random factor is nested within former random factor. The three-way interaction in M1 was removed because this interaction was mainly due to one data point having very high leverage (see text and Fig. S1)

\begin{tabular}{|c|c|c|c|c|c|c|c|c|}
\hline Model & Model type & $\begin{array}{l}\text { Random } \\
\text { factor }\end{array}$ & Response variable & Explanatory variables & $\mathrm{X}^{2}$ & Df & $\mathrm{AIC}$ & $P$ value \\
\hline \multirow[t]{7}{*}{ M1 } & \multirow{7}{*}{$\begin{array}{l}\text { GLMM } \\
\quad \text { (Poisson) }\end{array}$} & \multirow{7}{*}{$\begin{array}{l}\text { Day/ } \\
\text { Colony }\end{array}$} & \multirow{7}{*}{$\begin{array}{l}\text { P. larvae spore level } \\
\text { (CFU) }\end{array}$} & Quarantine status & 3.4 & 1 & 1376.3 & 0.06 \\
\hline & & & & Treatment & 0.0 & 1 & 1378.3 & 0.91 \\
\hline & & & & Diversity & 0.2 & 1 & 1380.1 & 0.64 \\
\hline & & & & Diversity $\times$ Quarantine status & 0.4 & 1 & 1381.6 & 0.48 \\
\hline & & & & Treatment $\times$ Diversity & 0.1 & 1 & 1383.5 & 0.74 \\
\hline & & & & Treatment $\times$ Quarantine status & 0.0 & 1 & 1385.8 & 0.92 \\
\hline & & & & Treatment $\times$ Diversity $\times$ Quarantine status & 3.0 & 1 & 1414.6 & $<0.001$ \\
\hline \multirow[t]{7}{*}{ M2 } & \multirow{7}{*}{$\begin{array}{l}\text { GLMM } \\
\text { (Poisson) }\end{array}$} & \multirow{7}{*}{$\begin{array}{l}\text { Day/ } \\
\text { Colony }\end{array}$} & \multirow{7}{*}{$\begin{array}{l}\text { P. larvae spore level } \\
(\mathrm{CFU})\end{array}$} & Quarantine status & 3.4 & 1 & 1376.3 & 0.06 \\
\hline & & & & Treatment & 0.0 & 1 & 1378.3 & 0.91 \\
\hline & & & & Cumulative abundance & 0.0 & 1 & 1380.3 & 0.93 \\
\hline & & & & Cumulative abundance $\times$ Quarantine status & 0.0 & 1 & 1382.3 & 0.88 \\
\hline & & & & Treatment $\times$ Cumulative abundance & 0.0 & 1 & 1384.3 & 0.94 \\
\hline & & & & Treatment $\times$ Quarantine status & 1.4 & 1 & 1385.8 & 0.22 \\
\hline & & & & $\begin{array}{l}\text { Treatment } \times \text { Cumulative abundance } \times \text { Quarantine } \\
\text { status }\end{array}$ & 24.3 & 1 & 1408.7 & 0.10 \\
\hline
\end{tabular}

investigated the effect of treatment on the diversity of all hbsLAB (Table 3: M4) and the abundance of individual hbs-LAB (Table 3: M5).

The third objective was to compare the two methods (MALDI-TOF MS and qPCR) for detection of hbs-LAB. These analyses only involved data for the eight hbs-LAB that where detectable by both methods, since L. kunkeei, $L$. apinorum, and L. mellifer could not be identified by qPCR due to the lack of specific primers and $B$. asteroides Bin2N and B. asteroides Bin $7 \mathrm{~N}$ could not be differentiated by MALDI-TOF MS. Firstly, we compared the number of hbsLAB detected in a sample by qPCR and MALDI-TOF MS (Table 4: M6). Secondly, the relationship of how often each
hbs-LAB was detected versus not being detected in all samples from all days was analyzed (Table 4: M7).

All univariate models consisted of generalized models (generalized linear model, GLM) and mixed models (linear mixed model, LMM; generalized linear mixed model, GLMM). Each model started with the main factors of interest and all interactions of interest following a backwards selection until the minimal adequate model was reached. Nonsignificant interactions/main effects, based on the Wald chisquare tests (Type III test) and an information criterion (AIC), were stepwise removed. Models with a Binomial response (sum of successes and failures of detection for both methods) were analyzed with logit link, a Poisson response with a log

Table 2 Multivariate analysis of co-variance (MANCOVA; Type III test) from multivariate linear model investigating the relationship between hbs-LAB quantified with qPCR. Non-significant terms (italicized) were removed stepwise from the final model starting from the bottom row

$P$. larvae, treatment and quarantine status, and the abundance of the ten

\begin{tabular}{|c|c|c|c|c|c|c|c|}
\hline Model & Model type & Response variable & Explanatory variables & $\begin{array}{l}\text { Pillai's } \\
\text { trace }\end{array}$ & $F$ & $\begin{array}{l}\text { Df (num, } \\
\text { den) }\end{array}$ & $\begin{array}{l}P \\
\text { value }\end{array}$ \\
\hline \multirow[t]{7}{*}{ M3 } & \multirow[t]{7}{*}{ MANCOVA } & \multirow{7}{*}{$\begin{array}{l}\text { Abundance (of each of the } 10 \\
\text { hbs-LAB) }\end{array}$} & Treatment & 0.02 & 0.74 & 10,273 & 0.68 \\
\hline & & & Quarantine status & 0.07 & 2.11 & 10,273 & 0.02 \\
\hline & & & Treatment $\times$ Quarantine status & 0.08 & 2.44 & 10,273 & $<0.01$ \\
\hline & & & P. larvae & 0.03 & 0.86 & 10,272 & 0.56 \\
\hline & & & P. larvae $\times$ Quarantine status & 0.02 & 0.77 & 10,271 & 0.64 \\
\hline & & & P. larvae $\times$ Treatment & 0.01 & 0.47 & 10,270 & 0.90 \\
\hline & & & $\begin{array}{l}\text { P. larvae } \times \text { Treatment } \times \text { Quarantine } \\
\text { status }\end{array}$ & 0.02 & 0.71 & 10,269 & 0.71 \\
\hline
\end{tabular}


Table 3 Analysis-of-deviance tables (Type III test) from linear mixed model (LMM) and generalized linear mixed model (GLMM) investigating the effect of hbs-LAB treatment on the hbs-LAB diversity in the honey crop (M4) and abundance of each hbs-LAB (M5). Non- significant terms (italicized) were removed stepwise from the final model starting from the bottom row. "/" means latter random factor is nested within former random factor

\begin{tabular}{|c|c|c|c|c|c|c|c|c|}
\hline Model & Model Type & Random factor & Response Variable & Explanatory Variables & $X^{2}$ & Df & AIC & $P$ value \\
\hline \multirow[t]{3}{*}{ M4 } & \multirow[t]{3}{*}{ LMM } & \multirow[t]{3}{*}{ Day } & \multirow[t]{3}{*}{ Diversity (Shannon) } & Quarantine status & 18.8 & 1 & 970.4 & $<0.001$ \\
\hline & & & & Treatment & 0.0 & 1 & 974.3 & 0.81 \\
\hline & & & & Treatment $\times$ Quarantine status & 0.0 & 1 & 976.8 & 0.83 \\
\hline \multirow[t]{7}{*}{ M5 } & \multirow[t]{7}{*}{ GLMM (Poisson) } & \multirow{7}{*}{$\begin{array}{l}\text { Day/ } \\
\text { Colony }\end{array}$} & \multirow[t]{7}{*}{ Individual abundance } & Strain & 230,430 & 9 & \multirow[t]{7}{*}{$1,424,487$} & $<0.001$ \\
\hline & & & & Treatment & 0.3 & 1 & & 0.57 \\
\hline & & & & Quarantine status & 6.3 & 1 & & 0.01 \\
\hline & & & & Strain $\times$ Treatment & 52,529 & 9 & & $<0.001$ \\
\hline & & & & Strain $\times$ Quarantine status & 17,599 & 9 & & $<0.001$ \\
\hline & & & & Treatment $\times$ Quarantine status & 4.7 & 1 & & 0.02 \\
\hline & & & & Strain $\times$ Treatment $\times$ Quarantine status & 41,315 & 9 & & $<0.001$ \\
\hline
\end{tabular}

link, and normal distribution with identity link function. Recommended visual inspection of residuals [44] necessitated a log-transformation in model 4 (Table 3: M4). In all mixed models, Gaussian random factors were included to model the hierarchical data structure; therefore, the repeated measure structure was accounted for. In the Binomial and Poisson models, every data pair/point received its own likelihood by nesting random factors until the observational level [45]. An autocorrelation for the sampling occasions was not included in the models because (i) preliminary analysis revealed very weak temporal correlation (e.g., M6 with covariance matrix with continuous autoregressive process: $\mathrm{Phi}=0.2$ ), (ii) quarantined and non-quarantined colonies should be similarly affected by time as both received either hbs-LAB treatments or placebo right after the sampling I and II, and (iii) there was no indication of a general temporal effect for any treatment-

Table 4 Analysis-of-deviance tables (Type III test) from generalized linear model (GLM) and generalized linear mixed model (GLMM) investigating the two methods (MALDI-TOF MS, qPCR) for detecting eight hbs-LAB that were available for both (see text). The number of different quarantine status combination (Fig. S3). The true sample size is 12 ( $n=12$ honeybee colonies) for each of the six sampling occasions $(12 \times 6=72$ samples per treatment-quarantine combination). However, three non-quarantined colonies (two treated with hbs-LAB and one treated with placebo) died before the last sampling occasion, decreasing the sample size slightly.

The complete analysis was conducted using $\mathrm{R}$ [46]. The functions lmer (LMM) and glmer (GLMM) from the package lme4 [47], Anova from the package car for the type III test [48], and the packages vegan [49], multcomp [50], and lsmeans [51] were used. The latter two packages were used to back-transform the response variables to linear scale (before calculating the arithmetic mean) and extract (Tukey method adjusted) pairwise comparisons of interest.

hbs-LAB detected (M6) and the relationship of detecting a hbs-LAB or not finding it (M7) were compared. Non-significant terms (italicized) were removed stepwise from the final model starting from the bottom row. " " means latter random factor is nested within former random factor

\begin{tabular}{|c|c|c|c|c|c|c|c|c|}
\hline Model & Model type & $\begin{array}{l}\text { Random } \\
\text { factor }\end{array}$ & Response variable & Explanatory variables & $X^{2}$ & Df & AIC & $P$ value \\
\hline \multirow[t]{7}{*}{ M6 } & \multirow{7}{*}{$\begin{array}{l}\text { GLMM } \\
\text { (Poisson) }\end{array}$} & \multirow{7}{*}{$\begin{array}{l}\text { Day/ } \\
\text { Colony }\end{array}$} & \multirow[t]{7}{*}{ Number of strains detected } & Method & 112.6 & 1 & 2241.8 & $<0.001$ \\
\hline & & & & Quarantine status & 23.8 & 1 & & $<0.001$ \\
\hline & & & & Treatment & 4.4 & 1 & & 0.03 \\
\hline & & & & Method $\times$ Treatment & 4.0 & 1 & & 0.04 \\
\hline & & & & Method $\times$ Quarantine status & 0.0 & 1 & 2243.8 & 0.95 \\
\hline & & & & Treatment $\times$ Quarantine status & 0.2 & 1 & 2245.6 & 0.62 \\
\hline & & & & $\begin{array}{l}\text { Method } \times \text { Treatment } \times \text { Quarantine } \\
\text { status }\end{array}$ & 0.9 & 1 & 2246.7 & 0.33 \\
\hline \multirow[t]{3}{*}{ M7 } & \multirow{3}{*}{$\begin{array}{l}\text { GLM } \\
\text { (binomial) }\end{array}$} & \multirow[t]{3}{*}{-} & \multirow{3}{*}{$\begin{array}{l}\text { Strain detection probability (failures, } \\
\text { successes) }\end{array}$} & Strain & 206.7 & 7 & 123.9 & $<0.001$ \\
\hline & & & & Method & 44.0 & 1 & & $<0.001$ \\
\hline & & & & Strain $\times$ Method & 140.4 & 7 & & $<0.001$ \\
\hline
\end{tabular}




\section{Results}

\section{The Effect of Oral Hbs-LAB Administration on Colony-Level Hbs-LAB Composition, P. larvae Prevalence, and their Potential Interaction}

Neither treatment with supplemental hbs-LAB (Table 1: M1 and M2, Fig. 1A), nor the hbs-LAB diversity (Table 1: M1, Fig. S1), nor the cumulative abundance of all hbs-LAB in the honey crop (Table 1: M2), nor the interaction between treatment and diversity/abundance (Table 1: M1 and M2) had an effect on the $P$. larvae spore levels. However, we found a strong tendency for higher P. larvae spore levels in quarantined colonies (Fig. 1B). At the beginning of model selection, we found an interaction between supplemental treatment, hbs-LAB diversity (Shannon index), and AFB quarantine status. However, this interaction was mainly due to one data point (Fig. S1) having very high leverage in the model. This interaction term was therefore removed, which considerably improved the model parsimony (AIC dropped by 28.8 points), justifying the removal. The subsequent selected model revealed no interactions.

In order to investigate the combined relationship between the various hbs-LAB and P. larvae, a multivariate model was run using the abundances of each individual hbs-LAB as multivariate response variables (Table 2 : M3). This showed that the abundance of all hbs-LAB combined was affected by an interaction between the supplemental hbs-LAB treatment and the AFB quarantine status of the apiaries. However, investigating the univariate relationship of individual hbs-LAB abundances within the model showed no interaction effect between treatment and quarantine status, no effect of treatment and an effect of quarantine status for all individual hbs-LAB abundances except for L. kimbladii Hma2N and B. asteroides Bin2N (Table S1). Furthermore, no relationship between the abundance of any individual hbs-LAB and $P$. larvae spore levels was noticed.
The Effect of Oral Hbs-LAB Administration on Hbs-LAB Diversity and Individual Abundance in the Honey Crop

The diversity of hbs-LAB in the honey crop was unaffected by supplemental treatment in either the quarantined and nonquarantined apiaries, but we found significantly higher diversity (around 19\%) in quarantined colonies (Table 3: M4; Fig. 2; see also Fig. S2 for variability of diversity over time with regard to treatment and quarantine status).

Only weak support was observed for an effect of supplementation of hbs-LAB on changes in the abundance of individual hbs-LAB (Fig. 3). Since the model showed a significant three-way interaction between strain, treatment, and quarantine status (Table 3: M5), we extracted the comparisons of interest (treatment-quarantine status combinations within each hbs-LAB) from all 780 comparisons. Only in one case (quarantined: B. asteroides Bin $2 \mathrm{~N}$ ) we found a higher abundance in the hbs-LAB treatment. Further illustrating the absence of a supplementation-abundance relationship is the fact that in two cases (non-quarantined: L. kullabergensis Biut2N, B. coryneforme Bma6N), we observed a higher abundance in the placebo treatment. However, we found strong support for higher hbs-LAB abundance if colonies had a history of infection (quarantined colonies). For example, of the 20 comparisons (10 strains, 2 treatments), 14 showed significantly higher abundance if the colony was quarantined.

\section{Comparison of MALDI-TOF MS and qPCR for Detecting Individual Hbs-LAB}

Based on the number of detecting or not detecting different hbs-LAB, we could compare the two most common techniques, qPCR and MALDI-TOF MS for detection and identification of these bacteria using eight strains that were detectable with both methods. The number of hbs-LAB detected in a sample was affected by the method-treatment interaction while all other
Fig. 1 Abundance of P. larvaecolony forming units (CFU) found in samples from colonies regarding treatments $(\mathbf{A})$ and/or quarantine status $(\mathbf{B})$. The squares show the predicted marginal means with confidence limits from the statistical model. The gray dots (with a separate scale in gray on the right side) show the original data ( $\log 10$ transformed after addition of one to show zero values). The hash mark indicates as strong tendency of higher $P$. larvae spore levels in quarantined colonies $(P=0.06)$

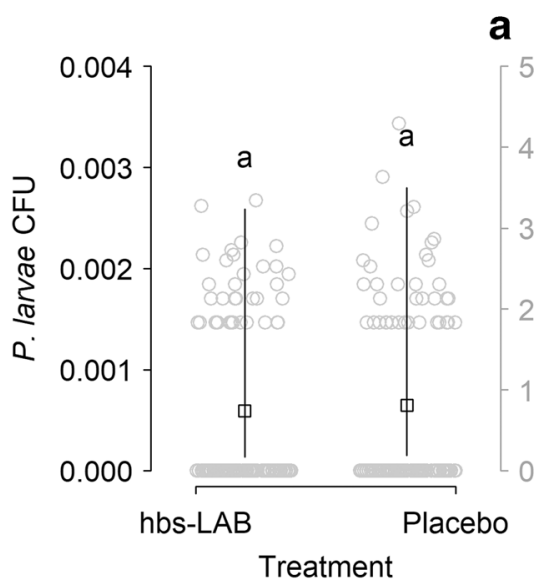

a

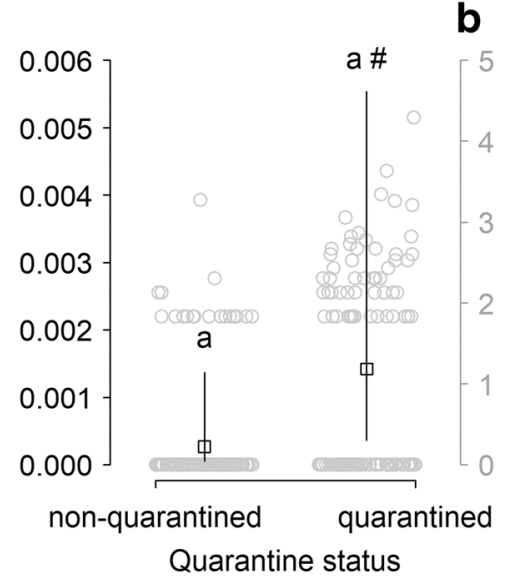


Fig. 2 Hbs-LAB diversity (Shannon index) found in samples from colonies regarding treatments $(\mathbf{A})$ or quarantine status (B). Different lower-case letters indicate significant differences between predicted marginal means with confidence limits while gray dots represent the original diversity of all sampling occasions of all colonies

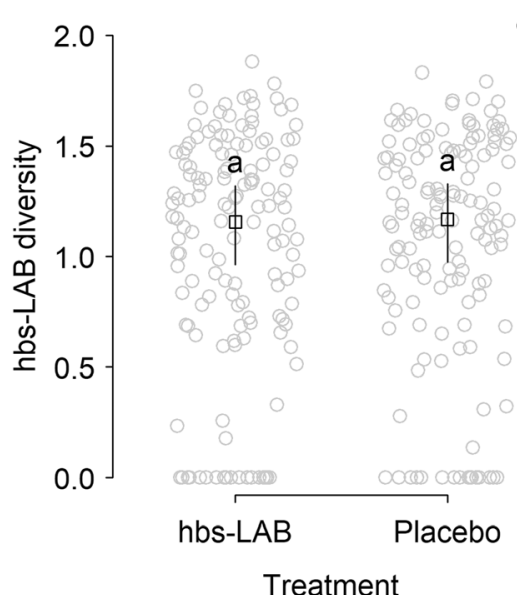

a

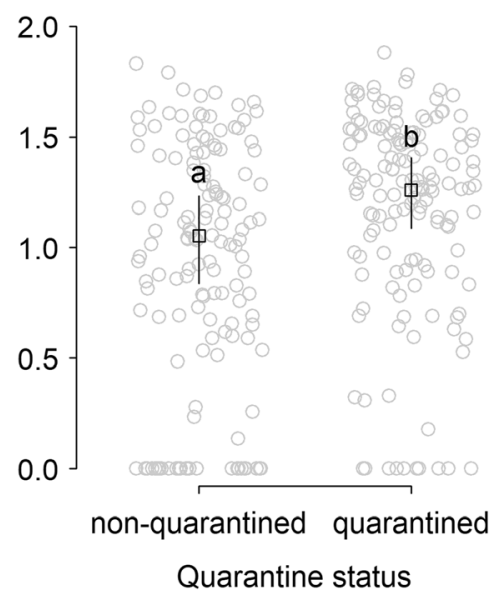

interactions between the method, the treatment, and the quarantine status were not significant (Table 4: M6). However, pairwise comparisons of the mean values did not show differences between treatments, and regardless of quarantine status more hbs-LAB could be detected using qPCR (Fig. 4A). Moreover, we detected generally more hbs-LAB in the quarantined colonies independent of the method (Fig. 4B).

Investigating how often each particular hbs-LAB was detected or not in all samples showed that seven out of eight hbsLAB had higher detection probability using qPCR compared to MALDI-TOF MS. The model resulted in an interaction between strains and method, which can be explained by the different detection probabilities with the two methods (Table 4: M7; Fig. 4C; see Table S2 for comparisons among all method-strains combinations). The only hbs-LAB that was not more efficiently detected by qPCR, was $L$. kimbladii (Hma2N); in this case both techniques were equally good. The greatest difference was found for $L$. apis (Hma11N) that was detected up to approx. sevenfold more often than not being detected (249 times detected /37 times not detected; Fig. 4C), which represented an approx. 12-fold better detection with qPCR compared to MALDI-TOF MS ((249/37)/(99/187)).

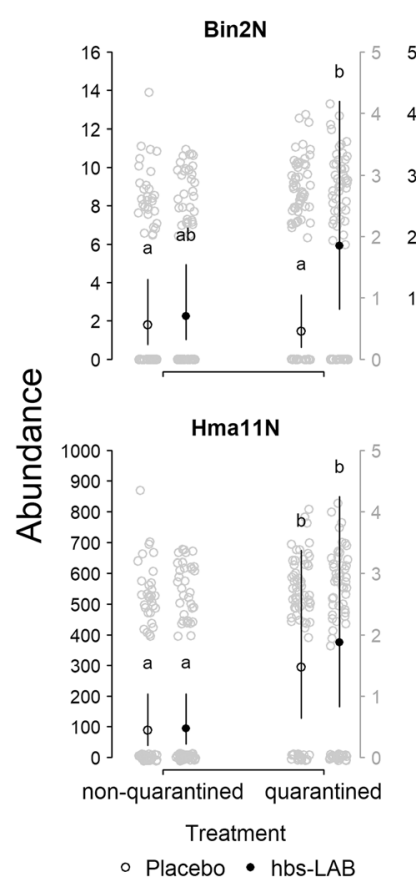

Fig. 3 Abundance of each hbs-LAB found in a sample with qPCR. The black dots show the predicted marginal means with confidence limits from the statistical model. The gray dots (with a separate scale in gray on the right side) show the original data ( $\log 10$ transformed after addition

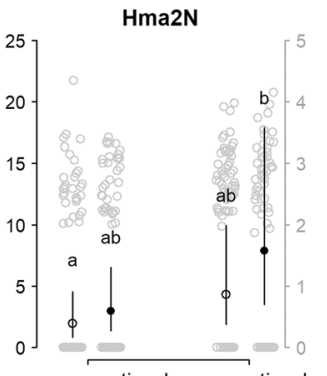

Biut2N
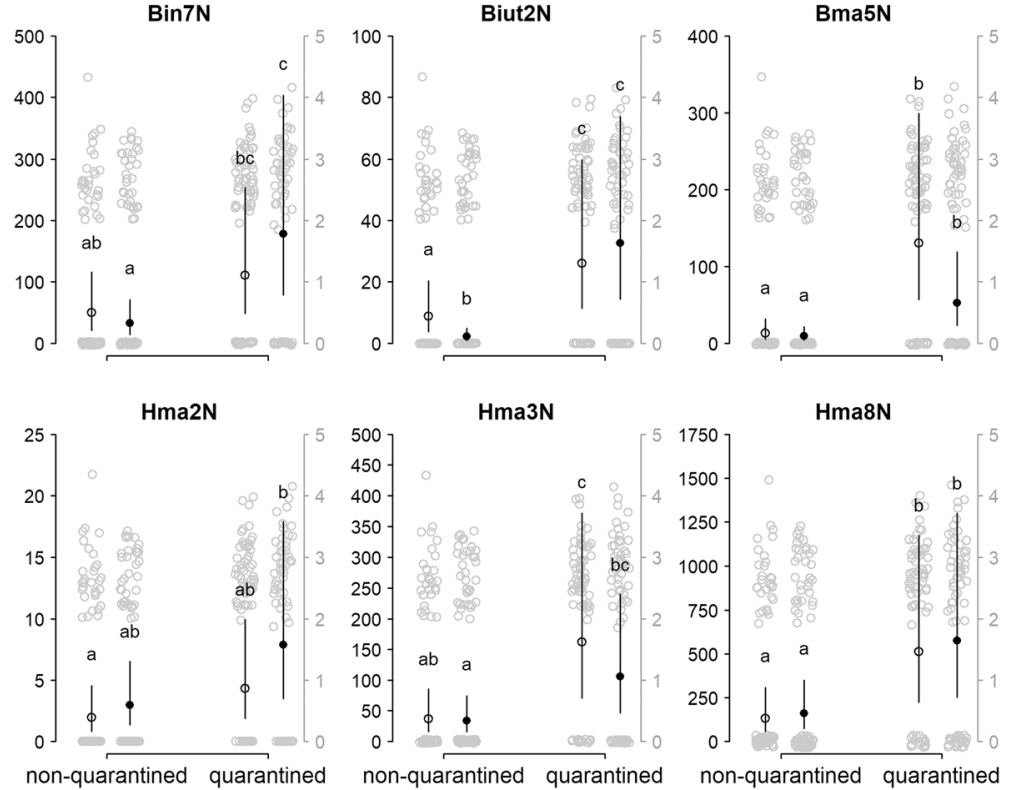

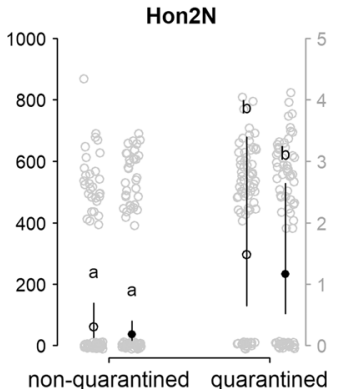

Bma5N
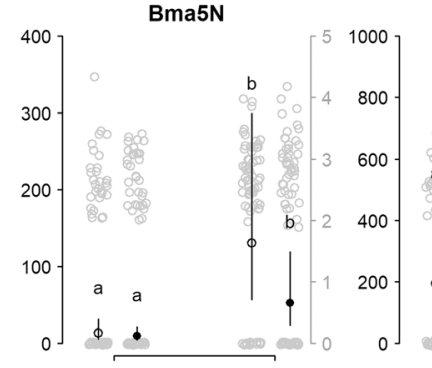

Bma6N

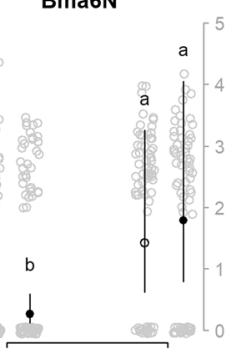

Hon2N

non-quarantined quarantined

\section{Quarantine status}

of one to show zero values). Different letters indicate significant differences between treatments-quarantine status combinations within each hbs-LAB 


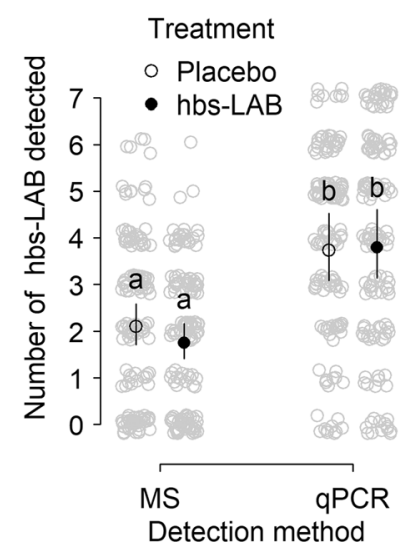

a

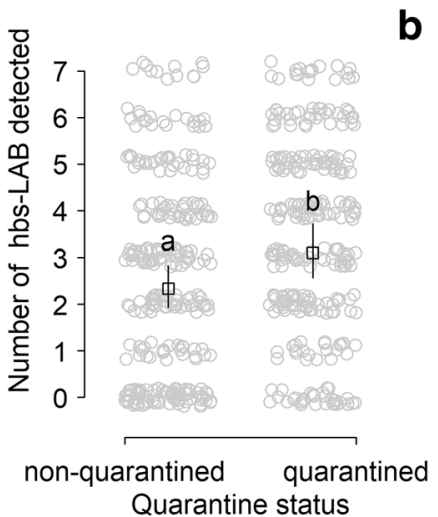

Fig. 4 The number of different hbs-LAB found in a sample was greater using qPCR technique (A) and in the quarantined honeybee colonies (B). Individual hbs-LAB was significantly better detected using qPCR in comparison with MALDI-TOF MS, except Hma2N that was detected similarly with both methods $(\mathbf{C})$. Different lower-case letters indicate significant differences between predicted marginal means with confidence limits form the respective models. In $\mathbf{A}$, all four combinations are

\section{Discussion}

Bacteria in the genera Lactobacillus and Bifidobacterium produce a range of antimicrobial metabolites, such as organic acids, antimicrobial peptides, and bacteriocins [17, 52-54]. It is therefore not surprising that their potential for supporting honeybee health, by limiting the virulence and spread of pathogens, has received much attention. However, only very few relevant field studies have been conducted on the effects of feed supplements containing beneficial bacteria on honeybee colony health and performance $[12,17,28,30]$.

In our experiments we showed that feed supplements containing hbs-LAB had no effect on hbs-LAB composition and abundance in the honey crop. We further verified our previous finding that $P$. larvae spore levels in the colonies are not altered by administration of hbs-LAB [30]. However, we did observe that hbs-LAB diversity ( $+19 \%$ Shannon index, higher number of strains) was much higher in quarantined colonies (i.e., those with a history of an AFB outbreak) than in non-quarantined colonies (i.e., those without a history of AFB) although hbs-
C

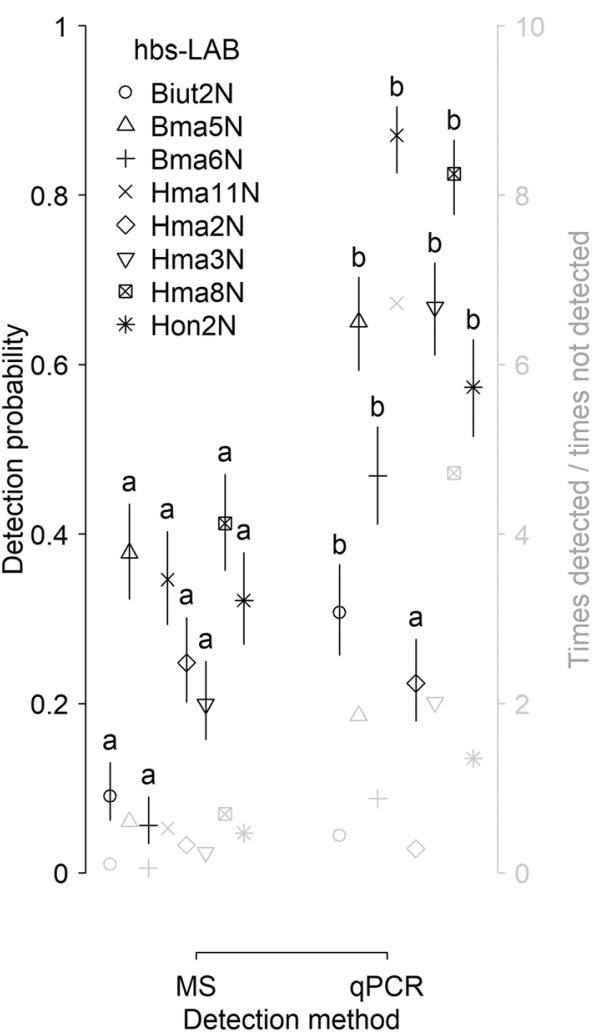

compared regardless of quarantine status; in $\mathbf{B}$, quarantine status is compared regardless of treatment-methods, and in $\mathbf{C}$, different methods are compared for each hbs-LAB respectively. In $\mathbf{A}$ and $\mathbf{B}$, the gray circles show the original data (all colonies at all sampling occasions) while in $\mathbf{C}$, the gray symbols show the original ratio of detection (times detected divided by times not detected)

LAB diversity was otherwise unrelated to $P$. larvae spore levels. This can have several possible explanations. Since the observations are coincidental with quarantine status, they could be related to any systematic difference between the colonies in the two sets of apiaries. This includes not only AFB history and P. larvae spore levels but also differences in the age of the bee equipment, colony establishment in 2015, geography and microclimate, different foraging resources, etc. Excluding all these alternatives, will require dedicated experimentation. However, qualitative and quantitative changes in the honeybee symbiotic microbiome due to pathogen pressure has been reported previously, in relation to $P$. larvae infection [55], Melissococcus pluton (the infective agent of European foulbrood) [56] and Varroa destructor infestation [57, 58]. It has been shown that $B$. asteroides abundance decreased in symptomatic $P$. larvae-infected honeybee colonies [55], while the proportions of L. kunkeei, $B$. coryneforme, and L. mellis were higher in symptomatic M. -pluton infected honeybees [56]. Furthermore, the abundance of L. mellis, L. kunkeei, L. kullabergensis, 
L. helsingborgensis, and L. kimbladii increased with increasing levels of varroosis [57]. These results suggest that the hbs-LAB and composition in adult honeybees may be a factor in brood diseases, even if the adult honeybees themselves are not affected [59], either through altering the ability of adult bees to engage in social immune responses (hygienic behavior) or through changes in the bacterial composition driven by bacterial infections in the brood; acquired and transmitted by adult bees through hygienic behavior and social interactions. It is only in the larvae that $P$. larvae and hbs-LAB can directly influence each other. The initial composition of the hbs-LAB that newly hatched larvae are exposed to would reflect its composition in the adults. Any subsequent change in adult bee hbs-LAB composition due to P. larvae infection in the larvae would have to be filtered back to the adult bee population through hygienic behavior and other interactions between adults and larvae, in order to affect the hbsLAB composition at the colony level. There are a number of ways by which this could theoretically happen, but these are indirect and long-term, possibly involving a combination of innate larval immunity, adult hygienic behavior and colony-level population turnover. Furthermore, hbs-LAB composition changes during the season, depending on the flowers visited and the honeybees' health status [20]. These bacteria produce different metabolites such as organic acids [22], peptides, and proteins that are species or even strain-specific [23]. For example, L. kunkeei is the most dominant hbs-LAB isolated from honey crops, honey, pollen, and beebread during spring and summer [20, $31,56,60]$, but is practically absent during the winter. It has been noticed that $L$. kunkeei produces a more diverse range of unknown proteins with putative antimicrobial activities than the other hbs-LAB [23]. Furthermore, we previously showed that hbs-LAB produce antimicrobial substances, which can inhibit the growth of $P$. larvae spores and vegetative cells at the individual level [29]. We can therefore not exclude the possibility that administration of one highly potential hbs-LAB may have a practically relevant effect in form of lowering $P$. larvae spore levels. It can, however, be debated whether supplemental administration of hbs-LAB can ever be effective for managing pathogens at the colony level given what we have shown here and previously [30], i.e., that these effects do not occur at the colony level. Hence, any promising results of the effects of hbs-LAB on pathogens at the individual level in controlled laboratory experiments [18] do not necessarily translate directly into an effective treatment for honeybee colonies.

The absence of a colony-wide effect could be explained by multiple reasons. In previous studies, an inverse relationship between the individual- and colony-level virulence for the two principal genotypes of $P$. larvae has been observed. The more virulent genotype at the individual level (ERIC-II) kills the larvae before they are capped, such that they can be easily detected and removed by social behaviors resulting in a reduced virulence at the colony level. The reverse is true for the ERIC-I genotype, whose reduced virulence at an individual level allows infected larvae to be capped, escaping early detection and removal, such that the infection finishes with spore production in hard sticky scales and thus a higher virulence at colony level [61]. By similar reasoning, those larvae with strong individual immune defenses that delay infection may therefore compromise the efficacy of the social defenses relative to larvae with weaker individual immunity [25]. Moreover, honeybee colonies have multiple homeostatic mechanisms for managing colony health and performance, of which, hbs-LAB are a small component. The close hbsLAB-host symbiotic relationship is naturally recalcitrant to any outside efforts at manipulating either the abundance or the composition of the hbs-LAB. The contrasting results between individual and colony level experimentation highlight the strength of the honeybee homeostatic mechanisms in neutralizing the effects of any attempt to manipulate the natural conditions of the nest $[62,63]$. Other studies have also shown that using beneficial microbes for treatment does not have the expected positive effects [17, 28, 64]. For example, Maggi et al. showed that supplying colonies with organic acids produced by the bacterium Lactobacillus johnsonii CRL1647 did not change the disease dynamics of Nosema spp. at the colony level [17]. Pretreatment with Snodgrassella alvi made honeybees more susceptible to the protozoan Lotmaria passim, suggesting that the probiotic therapy has complicated consequences for parasite susceptibility, microbiota homeostasis, host developmental, and detoxification response pathways [64]. Ptaszyńska et al. revealed that supplementing honeybee diets solely with the commercial probiotic, Lactobacillus rhamnosus with/without the prebiotic inulin, increased mortality levels in Nosema ceranae-infected honeybees [28]. It was recently shown that hbs-LAB may have short-term negative effects on the brood [30] although no negative effects of oral hbs-LAB administration like increased susceptibility (P. larvae spore counts higher in the hbs-LAB treatment) or elevated mortality among the quarantined colonies could be observed in this study. Whether more frequent hbs-LAB administration or higher dosages would have made much difference need further studies [27].

The comparison of the two different methods for detecting, identifying, and quantifying hbs-LAB (MALDI-TOF MS and qPCR) showed that qPCR could detect a wider range of hbsLAB, with higher specificity and sensitivity than MALDITOF MS, although in specific cases, MALDI-TOF could differentiate hbs-LAB (e.g., L. kunkeei, L. apinorum, and L. mellifer) that qPCR could not. It should be noted that one of the drawbacks of qPCR assays of bacterial DNA is that it does not distinguish between dead and alive cells, meaning 
that the bacterial composition measured by qPCR may not completely reflect the living community. Using bacterial mRNA as an (RT)-qPCR template allows for only alive cells to be assessed, but is less reliable for quantifying the number of CFU, due to the confounding effect of differential RNA expression levels on cellular quantification. Although MALDI-TOF MS itself is relatively simple, quick, and cheap to perform, it requires that the bacteria can be cultured on laboratory media prior to analysis, which may differ for different types of bacteria. Consequently, also analysis by MALDI-TOF MS may not completely reflect the living community. Moreover, MALDI-TOF MS still requires supplementary methods, such as $16 \mathrm{~S}$ rRNA sequencing, to differentiate closely related species or strains [65]. In this study, two closely related strains of B. asteroides (Bin2N and Bin $7 \mathrm{~N}$ ) that could be differentiated by qPCR were indistinguishable using MALDI-TOF MS. Since all methods have their biases, a fully comprehensive analysis of (hbs-LAB) microbial communities should therefore use multiple complementary methodologies [2].

In conclusion, we showed that the hbs-LAB composition of honeybees is more diverse in honeybee colonies with elevated $P$. larvae spore levels, but we found no support that the hbs-LAB composition in bees can be altered by this type or dose of hbs-LAB supplements. This work does not refute the beneficial nature of hbs-LAB found in the honey stomachs of honeybees, as abundantly shown previously $[18,19,22,31]$. However, we did not find any effect of similar supplementary hbs-LAB administration on the $P$. larvae spore levels at colony level, highlighting that data and conclusions derived from laboratory experiments on individual bees may not be applicable for the colony $[2,27]$.

Acknowledgments The funders had no role in study design, data collection, and interpretation, or the decision to submit the work for publication. ConCellae $\mathrm{AB}$ are the producers of SymBeeotic ${ }^{\mathrm{TM}}$, a proprietary blend of hbs-LAB for probiotic use in honeybee colonies, and IP rights holders of the PCR primer sequences that were used for the specific detection of different LAB species and isolates. The authors wish to thank Emmy Sundström, Emilia Semberg and Anette Mårtensson for technical assistance and Alejandra Vásquez and Tobias Olofsson for their contributions. Companies and their trademarked products are mentioned for scientific accuracy only and do not imply any specific endorsement or non-endorsement.

Authors' Contributions EF, JM and SL conceived and designed the experiments; SL performed the experiments; JS analyzed the data; KR and $\mathrm{BN}$ contributed reagents/materials/analysis tools; SS developed the qPCR assays; SL, JS, JM, EF, wrote the paper with editorial advice from KR and BN. All authors read and approved the paper.

Funding Information Open access funding provided by Swedish University of Agricultural Sciences. This research was supported by grant Dnr 222-2013-423 from the Swedish Research Council for Sustainable Development (FORMAS) to the Swedish University of Agricultural Sciences.
Data Availability Statements The datasets generated during and/or analyzed during the current study are available from the corresponding author on reasonable request.

Conflict of Interest The authors declare that they have no conflict of interest.

Open Access This article is distributed under the terms of the Creative Commons Attribution 4.0 International License (http:// creativecommons.org/licenses/by/4.0/), which permits unrestricted use, distribution, and reproduction in any medium, provided you give appropriate credit to the original author(s) and the source, provide a link to the Creative Commons license, and indicate if changes were made.

\section{References}

1. Cox-Foster DL, Conlan S, Holmes EC, Palacios G, Evans JD, Moran NA, Quan PL, Briese T, Hornig M, Geiser DM, Martinson V, vanEngelsdorp D, Kalkstein AL, Drysdale A, Hui J, Zhai J, Cui L, Hutchison SK, Simons JF, Egholm M, Pettis JS, Lipkin WI (2007) A metagenomic survey of microbes in honey bee colony collapse disorder. Science 318:283-287. https://doi.org/10.1126/ science. 1146498

2. Cornman RS, Dainat J, De MJR et al (2016) The bee microbiome: impact on bee health and model for evolution and ecology of hostmicrobe interactions. MBio 7:1-9. https://doi.org/10.1128/mBio. 02164-15.Invited

3. Corby-Harris V, Maes P, Anderson KE (2014) The bacterial communities associated with honey bee (Apis mellifera) foragers. PLoS One 9:e95056. https://doi.org/10.1371/journal.pone.0095056

4. Genersch E (2010) American foulbrood in honeybees and its causative agent, Paenibacillus larvae. J Invertebr Pathol 103:S10-S19. https://doi.org/10.1016/j.jip.2009.06.015

5. Genersch E, Forsgren E, Pentikäinen J et al (2006) Reclassification of Paenibacillus larvae subsp. pulvifaciens and Paenibacillus larvae subsp. larvae as Paenibacillus larvae without subspecies differentiation. Int J Syst Evol Microbiol 56:501-511. https://doi. org/10.1099/ijs.0.63928-0

6. Genersch E, Otte C (2003) The use of repetitive element PCR fingerprinting (rep-PCR) for genetic subtyping of German field isolates of Paenibacillus larvae subsp. larvae. Apidologie 34: 195-206

7. Bailey L, Ball B (1991) Honey Bee Pathology, 2nd ed. Elsevier. https://doi.org/10.1016/C2009-0-02695-6

8. Lindström A, Korpela S, Fries I (2008) The distribution of Paenibacillus larvae spores in adult bees and honey and larval mortality, following the addition of American foulbrood diseased brood or spore-contaminated honey in honey bee (Apis mellifera) colonies. J Invertebr Pathol 99:82-86

9. Fries I, Camazine S (2001) Implications of horizontal and vertical pathogen transmission for honey bee epidemiology. Apidologie 32: 199-214. https://doi.org/10.1051/apido:2001122

10. Elzen PJ, Westervelt D, Causey D, Ellis J, Hepburn HR, Neumann P (2002) Method of application of tylosin, an antibiotic for American foulbrood control, with effects on small hive beetle (Coleoptera: Nitidulidae) populations. J Econ Entomol 95:1119 1122. https://doi.org/10.1603/0022-0493-95.6.1119

11. Evans JD (2003) Diverse origins of tetracycline resistance in the honey bee bacterial pathogen Paenibacillus larvae. J Invertebr Pathol 83:46-50

12. Pătruică S, Mot D, Pătruică S, Mot D (2012) The effect of using prebiotic and probiotic products on intestinal micro-flora of the 
honeybee (Apis mellifera carpatica). Bull Entomol Res 102:1-5. https://doi.org/10.1017/S0007485312000144

13. Sabaté DC, Cruz MS, Benítez-Ahrendts MR, Audisio MC (2012) Beneficial effects of Bacillus subtilis subsp. subtilis Mori2, a honey-associated strain, on honeybee Colony performance. Probiotics Antimicrob Proteins 4:39-46. https://doi.org/10.1007/ s12602-011-9089-0

14. Janashia I, Alaux CC (2016) Specific immune stimulation by endogenous bacteria in honey bees (Hymenoptera: Apidae). J Econ Entomol 109:1474-1477. https://doi.org/10.1093/jee/tow065

15. Pătruică S, Huțu I (2013) Economic benefits of using prebiotic and probiotic products as supplements in stimulation feeds administered to bee colonies. Turk J Vet Anim Sci 37:259-263

16. Evans JD, Lopez DL (2004) Bacterial probiotics induce an immune response in the honey bee (Hymenoptera: Apidae). J Econ Entomol 97:752-756

17. Maggi M, Negri P, Plischuk S, Szawarski N, de Piano F, de Feudis L, Eguaras M, Audisio C (2013) Effects of the organic acids produced by a lactic acid bacterium in Apis mellifera colony development, Nosema ceranae control and fumagillin efficiency. Vet Microbiol 167:474-483. https://doi.org/10.1016/j.vetmic.2013.07. 030

18. Forsgren E, Olofsson TC, Vásquez A, Fries I (2010) Novel lactic acid bacteria inhibiting Paenibacillus larvae in honey bee larvae. Apidologie 41:99-108. https://doi.org/10.1051/apido/2009065

19. Killer J, Dubná S, Sedláček I, Švec P (2014) Lactobacillus apis sp. nov., from the stomach of honeybees (Apis mellifera), having an in vitro inhibitory effect on the causative agents of American and European foulbrood. Int J Syst Evol Microbiol 64:152-157. https:// doi.org/10.1099/ijs.0.053033-0

20. Olofsson TC, Vásquez A (2008) Detection and identification of a novel lactic acid bacterial flora within the honey stomach of the honeybee Apis mellifera. Curr Microbiol 57:356-363. https://doi. org/10.1007/s00284-008-9202-0

21. Olofsson TC, Alsterfjord M, Nilson B, Butler E, Vasquez A (2014) Lactobacillus apinorum sp. nov., Lactobacillus mellifer sp. nov., Lactobacillus mellis sp. nov., Lactobacillus melliventris sp. nov., Lactobacillus kimbladii sp. nov., Lactobacillus helsingborgensis sp. nov. and Lactobacillus kullabergensis sp. nov., isol. Int J Syst Evol Microbiol 64:3109-3119. https://doi.org/10.1099/ijs.0. 059600-0

22. Olofsson TC, Butler È, Markowicz P et al (2016) Lactic acid bacterial symbionts in honeybees - an unknown key to honey's antimicrobial and therapeutic activities. Int Wound J 13:668-679. https:// doi.org/10.1111/iwj.12345

23. Butler È, Alsterfjord M, Olofsson TC, Karlsson C, Malmström J, Vásquez A (2013) Proteins of novel lactic acid bacteria from Apis mellifera mellifera: an insight into the production of known extracellular proteins during microbial stress. BMC Microbiol 13:235

24. Rauch S, Ashiralieva A, Hedtke K, Genersch E (2009) Negative correlation between individual-insect-level virulence and colonylevel virulence of Paenibacillus larvae, the etiological agent of american foulbrood of honeybees. Appl Environ Microbiol 75: 3344-3347. https://doi.org/10.1128/AEM.02839-08

25. Evans JD, Aronstein K, Chen YP, Hetru C, Imler JL, Jiang H, Kanost M, Thompson GJ, Zou Z, Hultmark D (2006) Immune pathways and defence mechanisms in honey bees Apis mellifera. Insect Mol Biol 15:645-656

26. Evans JD, Spivak M (2010) Socialized medicine: individual and communal disease barriers in honey bees. J Invertebr Pathol 103: S62-S72. https://doi.org/10.1016/j.jip.2009.06.019

27. Alberoni D, Gaggìa F, Baffoni L, Di Gioia D (2016) Beneficial microorganisms for honey bees: problems and progresses. Appl Microbiol Biotechnol 100:9469-9482. https://doi.org/10.1007/ s00253-016-7870-4
28. Ptaszynska AA, Borsuk G, Zdybicka-Barabas A et al (2016) Are commercial probiotics and prebiotics effective in the treatment and prevention of honeybee nosemosis C? Parasitol Res 115:397-406. https://doi.org/10.1007/s00436-015-4761-z

29. Lamei S, Stephan JG, Riesbeck K, Vasquez A, Olofsson T, Nilson B, de Miranda JR, Forsgren E (2019) The secretome of honey beespecific lactic acid bacteria inhibits Paenibacillus larvae growth. J Apic Res 58:405-412. https://doi.org/10.1080/00218839.2019. 1572096

30. Stephan JG, Lamei S, Pettis JS, Riesbeck K, de Miranda JR, Forsgren E (2019) Honeybee-specific lactic acid bacterium supplements have no effect on American foulbrood-infected honeybee colonies. Appl Environ Microbiol 85:1-12. https://doi.org/10. 1128/AEM.00606-19

31. Vásquez A, Forsgren E, Fries I, Paxton RJ, Flaberg E, Szekely L, Olofsson TC (2012) Symbionts as major modulators of insect health: lactic acid bacteria and honeybees. PLoS One 7:e33188. https://doi.org/10.1371/journal.pone.0033188

32. Locke B, Low M, Forsgren E (2019) An integrated management strategy to prevent outbreaks and eliminate infection pressure of American foulbrood disease in a commercial beekeeping operation. Prev Vet Med 167:48-52. https://doi.org/10.1016/j.prevetmed. 2019.03.023

33. Nordström S, Fries I (1995) A comparison of media and cultural conditions for identification of Bacillus larvae in honey. J Apic Res 34:97-103

34. Lindström A, Fries I (2005) Sampling of adult bees for detection of American foulbrood (Paenibacillus larvae subsp larvae) spores in honey bee (Apis mellifera) colonies. J Apic Res 44:82-86. https:// doi.org/10.1080/00218839.2005.11101154

35. Lamei S, Hu YOO, Olofsson TC, Andersson AF, Forsgren E, Vásquez A (2017) Improvement of identification methods for honeybee specific lactic acid bacteria; future approaches. PLoS One 12: 1-12. https://doi.org/10.1371/journal.pone.0174614

36. Carreck NL, Andree M, Brent CS, Cox-Foster D, Dade HA, Ellis JD, Hatjina F, van Englesdorp D (2013) Standard methods for Apis mellifera anatomy and dissection. J Apic Res 52:1-40. https://doi. org/10.3896/IBRA.1.52.4.03

37. de Graaf DC, Alippi AM, Antúnez K, Aronstein KA, Budge G, de Koker D, de Smet L, Dingman DW, Evans JD, Foster LJ, Fünfhaus A, Garcia-Gonzalez E, Gregore A, Human H, Murray KD, Nguyen BK, Poppinga L, Spivak M, van Engelsdorp D, Wilkins S, Genersch E (2013) Standard methods for American foulbrood research. J Apic Res 52:1-28. https://doi.org/10.3896/IBRA.1.52.1. 11

38. Forsgren E, Laugen AT (2014) Prognostic value of using bee and hive debris samples for the detection of American foulbrood disease in honey bee colonies. Apidologie 45:10-20

39. Dobbelaere W, De Graaf D, Peeters J (2001) Development of a fast and reliable diagnostic method for American foulbrood disease (Paenibacillus larvae subs P. larvae) using a 16S rRNA gene based PCR. Apidologie 32:363-370

40. Nadkarni M, Martin FE, Jacques NA, Hunter N (2002) Determination of bacterial load by real-time PCR using a broad range (universal) probe and primer set. Microbiology 148:257-266

41. Butler E, Oien RF, Lindholm C, Olofsson TC, Nilson B, Vásquez A (2016) A pilot study investigating lactic acid bacterial symbionts from the honeybee in inhibiting human chronic wound pathogens. Int Wound J 13:729-737

42. Schielzeth $H$ (2010) Simple means to improve the interpretability of regression coefficients. Methods Ecol Evol 1:103-113. https://doi. org/10.1111/j.2041-210X.2010.00012.x

43. Everitt BS, Hothorn T (2014) A Handbook of Statistical Analyses using R. Chapman and Hall/CRC 
44. Zuur AAF, Ieno EENE, Elphick CCS (2010) A protocol for data exploration to avoid common statistical problems. Methods Ecol Evol 1:3-14. https://doi.org/10.1111/j.2041-210X.2009.00001.x

45. Harrison XA (2014) Using observation-level random effects to model overdispersion in count data in ecology and evolution. PeerJ 2:e616. https://doi.org/10.7717/peerj.616

46. R Core Team (2016) R: a language and environment for statistical computing. R Foundation for Statistical Computing, Vienna URL http://www.R-project.org/

47. Bates D, Mächler M, Bolker B, Walker S (2015) Fitting Linear Mixed-Effects Models Using lme4. J Stat Softw 67. https://doi. org/10.18637/jss.v067.i01

48. Fox J, Weisberg S (2019) An $\{\mathrm{R}\}$ Companion to Applied Regression. Sage Publications https://socialsciences.mcmaster.ca/ jfox/Books/Companion/. Accessed 10 Jan 2019

49. Oksanen J, Blanchet FG, Kindt R, Legendre P, McGlinn D, Minchin PR, O'Hara RB, Simpson GL, Solymos P, Henry M, Stevens H, Szoecs E, Wagner H (2019) Vegan: community ecology package. R package version 2.5-5. https://CRAN.R-project.org/ package=vegan. Accessed 10 Jan 2019

50. Hothorn T, Bretz F, Westfall P (2008) Simultaneous Inference in General Parametric Models. Biometrical J 50:346-363. https://doi. org/10.1002/bimj.200810425

51. Lenth RV (2016) Least-squares means: the R package lsmeans. J Stat Softw 69:1-33. https://doi.org/10.18637/jss.v069.i01

52. Billiet A, Meeus I, Cnockaert M, Vandamme P, van Oystaeyen A, Wäckers F, Smagghe G (2017) Effect of oral administration of lactic acid bacteria on colony performance and gut microbiota in indoor-reared bumblebees (Bombus terrestris). Apidologie 48:4150

53. Audisio MC (2016) Gram-positive Bacteria with probiotic potential for the Apis mellifera L. honey bee: the experience in the northwest of Argentina. Probiotics Antimicrob Proteins 9:22-31

54. Mudroňová $\mathrm{D}$, Toporčák J, Nemcová R, Gancarčíková S, Hajdučková V, Rumanovská K (2011) Lactobacillus sp. as a potential probiotic for the prevention of Paenibacillus larvae infection in honey bees. J Apic Res 50:323-324

55. Erban T, Ledvinka O, Kamler M, Nesvorna M, Hortova B, Tyl J, Titera D, Markovic M, Hubert J (2017) Honeybee (Apis mellifera)associated bacterial community affected by American foulbrood: detection of Paenibacillus larvae via microbiome analysis /631/ 158/855/631/326/2565/855/38/23/38/22/38/47 article. Sci Rep 7: 1-10. https://doi.org/10.1038/s41598-017-05076-8

56. Erban T, Ledvinka O, Kamler M, Hortova B, Nesvorna M, Tyl J, Titera D, Markovic M, Hubert J (2017) Bacterial community associated with worker honeybees ( Apis mellifera) affected by European foulbrood. PeerJ 5:e3816

57. Hubert J, Bicianova M, Ledvinka O et al (2016) Changes in the Bacteriome of honey bees associated with the parasite Varroa destructor, and pathogens Nosema and Lotmaria passim. Microb Ecol 73:685-698

58. Hubert J, Kamler M, Nesvorna M, Ledvinka O, Kopecky J, Erban T (2016) Comparison of Varroa destructor and worker honeybee microbiota within hives indicates shared bacteria. Microb Ecol 72: 448-459

59. Crailsheim K, Riessberger-Gallé U (2001) Honey bee agedependent resistance against American foulbrood. Apidologie 32: 91-103

60. Vásquez A, Olofsson TC (2009) The lactic acid bacteria involved in the production of bee pollen and bee bread. J Apic Res 48:189-195

61. Genersch E, Ashiralieva A, Fries I (2005) Strain- and genotypespecific differences in virulence of Paenibacillus larvae subsp. larvae, a bacterial pathogen causing American foulbrood disease in honeybees. Appl Environ Microbiol 71:7551-7555. https://doi.org/ 10.1128/AEM.71.11.7551-7555.2005

62. Liu F, He J, Fu W (2005) Highly controlled nest homeostasis of honey bees helps deactivate phenolics in nectar. Naturwissenschaften 92:297-299

63. Wu-Smart J, Spivak M (2016) Sub-lethal effects of dietary neonicotinoid insecticide exposure on honey bee queen fecundity and colony development. Sci Rep 6:32108

64. Schwarz RS, Moran NA, Evans JD (2016) Early gut colonizers shape parasite susceptibility and microbiota composition in honey bee workers. Proc Natl Acad Sci U S A 113:9345-9350. https://doi. org/10.1073/pnas.1606631113

65. Carbonnelle E, Mesquita C, Bille E, Day N, Dauphin B, Beretti JL, Ferroni A, Gutmann L, Nassif X (2011) MALDI-TOF mass spectrometry tools for bacterial identification in clinical microbiology laboratory. Clin Biochem 44:104-109 